\title{
Mixed Convection Flow Along Vertical Thin Needles Containing Gyrotactic Microorganism with Variable Heat, Mass and Motile Microorganism Flux
}

Nayema Islam Nima

University of Dhaka

Bader Alshuraiaan

Kuwait University

M. Ferdows ( $\square$ ferdows@du.ac.bd)

University of Dhaka

\section{Research Article}

Keywords: Mixed Convection Flow, gyrotactic microorganism, variable heat, mass and motile microorganism flux

Posted Date: July 29th, 2021

DOl: https://doi.org/10.21203/rs.3.rs-753328/v1

License: (c) (1) This work is licensed under a Creative Commons Attribution 4.0 International License. Read Full License 


\title{
Mixed Convection Flow along vertical thin needles containing gyrotactic microorganism with variable heat, mass and motile microorganism flux
}

\author{
Nayema Islam Nima ${ }^{1}$, Bader Alshuraiaan ${ }^{2}$ and M. Ferdows ${ }^{1, *}$ \\ ${ }^{1}$ Research Group of Fluid Flow Modeling and Simulation, Department of Applied Mathematics, \\ University of Dhaka, Dhaka-100, Bangladesh. \\ ${ }^{2}$ Department of Mechanical Engineering, Kuwait University, Safat 13060, Kuwait
}

\begin{abstract}
The problem of steady laminar mixed convection boundary layer flow along vertical thin needle with variable surface heat, mass and motile microorganism flux in the presence of gyrotactic microorganism is considered in this study. The dimensionless leading equations of continuity, momentum, concentraton and motile microorganism conservation are reduced to ordinary differential equations with the help of similarity transformations. The transformed governing equations are then numerically solved by using MATLAB BVP4C function. The research is reached to excellent argument by comparison in few cases between the results obtained from MATLAB and Maple algorithm with the help of dsolve command. Numerical calculations are carried out for various values of the dimensionless parameters of the problem which includes mixed convection parameter $\lambda$, power law index $\mathrm{m}$, buoyancy parameters $N_{1}, N_{2}$, Lewis parameter $\mathrm{Le}$, bioconvection lewis parameter $\mathrm{Lb}$, Bioconvection peclet number Pe and also the parameter a representing the needle size. It is also shown from the results that the surface (wall) temperature, surface fluid concentration, surface motile microorganism concentration and the corresponding velocity, temperature, concentration and motile microorganism profiles are significantly induced by these parameters. The results are pictured and discussed in detail.
\end{abstract}

\section{Introduction}

The study of mixed convection which is the combination of free and forced convection flow has become the great interest of many researchers over the last few decades because of its wide range of technological and industrial applications that have been reviewed in Refs. [1-3] such as heat exchanges placed in a low velocity environment, solar collectors exposed to wind currents, atmospheric boundary layer flows, nuclear reactors cooled during emergency shutdown and various electronic equipments. Several studies were done on mixed convection flow through different surfaces by many researchers such as Refs. [4-7]. Heat flux is another important phenomenon that can actually affect the heat process mechanism. Several studies in Refs. [8-13] are introduced on these topics and it has been shown that the fluid flow together with the uniform/ variable heat flux is very important in some of the manufacturing processes. Very recently Ibrahim [14] studied mixed convection nanofluid flow over non isothermal solid sphere with uniform heat flux and Ahmed [15] observed Magnetohyrodynamic flow for unsteady stretching sheet with variable surface heat flux. 
The study of microorganism has become greater attention of researchers to observe the development of microorganisms in bioconvection. Because of their mixing property and ability to improve mass transport, bioconvection phenomena have major applications in many biological systems and biotechnology. There are several notable applications in the fields of biomedicine (nanodrug delivery, cancer therapeutics), bio-microsystems (enzyme biosensors, biotechnology). Bioconvection is a development process in the field of fluid flow that works with the steps of selfpropelled up swimming microorganisms such as algae and bacteria that contain oxytaxis, gyrotaxis or gravitaxis organisms. Since motile microorganisms are denser than the liquid they are surrounded by, they swim upwards, resulting in different flow profiles in the system according to Refs.[16-20]. Gyrotaxis is most commonly found in a bottom-heavy microorganism. Kuznetsov [21] came up with the concept of putting motile microorganisms in nanofluid. Adding motile microorganisms to the suspension has many advantages, including better mass transfer, microscale mixing, and fluid stability. Significant papers on the application of bioconvection in thermobioconvection, microbial enhancement, bio-Microsystems, biofuels, and other bioengineering systems have been written in Refs.[ 22-29].Very recently Mahdy [30, 31] studied time-mixed convection nanofluid flow for rotating sphere and also Eyring -Powell nanofluid flow containing motile microorganisms. Waqas [32] observed Magneto-Burgers Nanofluid Stratified Flow with Swimming Motile Microorganisms and also bionanoconvective flow past a needle in the presence of Stefan blowing and gyrotactic microorganisms were observed by Amirsom [33] and Beg [34].

The flow over a thin needle has become a topic of interest for the researchers because of its importance in biomedical engineering where it arises in, for example cancer therapy and dermal administration of drugs. In order to measure the momentum and heat transfer behavior, flow analysis over such slender needles is also a major concern in experimental work. This occurs as a result of the needle's motion, which disrupts the free stream flow. As a result, this subject is of considerable practical importance, especially in the context of hot anemometers for finding wind's velocity, transportations, geothermal power generation, fiber technology, lubrications, aerospace, wire coating according to [35] and also in metal spinning and in micro/nanoscale equipment [36] including the removal of subterranean nuclear waste [37]. The thin needle is viewed as a slender body with the geometry having paraboloid of revolution where its thickness is smaller compared to the boundary layer thickness. Many researchers such as [38-44] have studied steady combined free and mixed convection boundary layer flow over a vertical thin needle. Grosan and Pop [45] pioneered the influence of nanoparticles on flow over a thin needle, which was later applied to a moving needle in a nanofluid by Soid et al. [46]. Hayat et al. [47] looked at the flow in a nanoliquid with a variable surface heat flux caused by a slender needle near a stagnation point. The Buongiorno mathematical model of nanofluid due to moving slender needle was used by Ahmad et al. [48]. Salleh et al. [49] then studied stability analysis to understand the additional impact of the heat source and chemical reaction in a nanofluid flow towards a slender needle. Very recently Waini et al. [50] and Khan et al. [51] studied hybrid nanofluid flow through the thin needle.

All studies mentioned above on free or mixed convection boundary layer flow over vertical thin needles refer to fixed needles immersed in a viscous and incompressible fluid. And have generally considered heat/mass transfer along either isothermal/non-isothermal or constant/variable wall heat flux needles. However, to the authors' best knowledge, the mixed convection boundary layer flow past a vertical thin needle containing gyrotactic microorganism with variable surface heat, 
mass and motile microorganism flux has not been reported in the literature, which motivates the present work. In view of the fact that this type of variable surface heat flux condition is commonly encountered in engineering practice, it merits separate attention.

To the best of my knowledge, the results obtained from the present study are new and original and have not been published elsewhere. Present study could be used in food and pharmaceutical industries, chemical processing equipment, fuel cell technology, enhanced oil recovery, etc.

\section{Model Formulation}

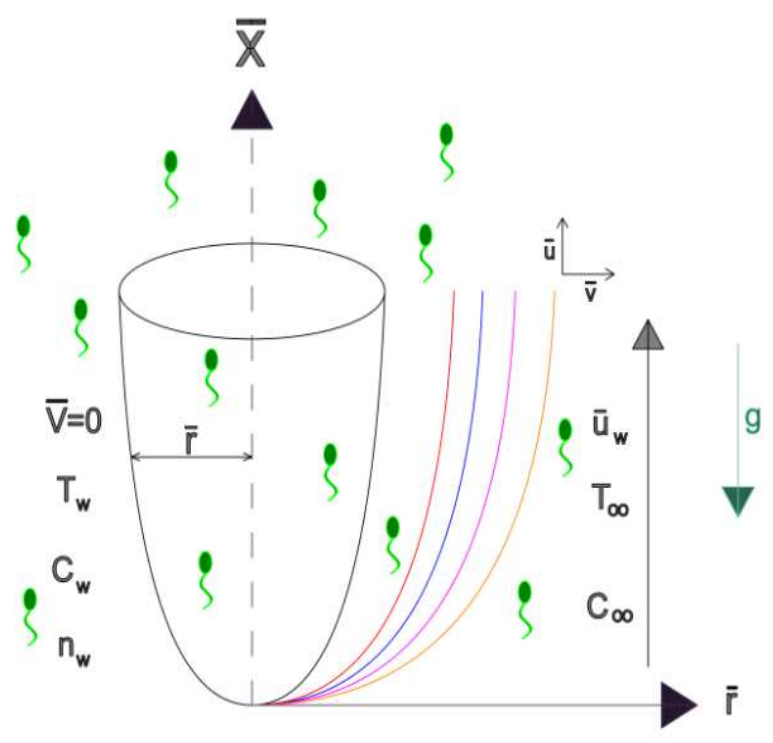

Fig 1. Flow model and coordinate system

2-D laminar free forced convective flow in the region external to a vertical thin needle with variable heat, mass and motile microorganism flux in the presence of gyrotactic microorganisms is considered. In fig 1. Flow model and system coordinates are shown where vertical slender needle whose radius is described by $\bar{r}=\bar{R}(\bar{x})$, where $\bar{x}$ and $\bar{r}$ are the axial and radial coordinates, respectively, with the $\bar{x}$-axis measures from the needle leading edge. The needle is considered thin when its thickness does not exceed that of boundary layer over it. $\bar{u}$ and $\bar{v}$ are the velocity components along the $\mathrm{x}$ and $\mathrm{r}$ axes. $U_{w}(\stackrel{\bar{x}}{x})$ is the velocity of vertical thin needle and $\mathrm{g}$ is the acceleration due to gravity. Following $[33,34,52,53]$ under the Boussinesq approximations the appropriate dimensionless equations in cylindrical coordinates are,

$$
\begin{aligned}
& \frac{1}{r} \frac{\partial^{2} \psi}{\partial r^{2}}=\lambda^{3}\left[\frac{\partial T}{\partial r}+N_{1} \frac{\partial C}{\partial r}+N_{2} \frac{\partial n}{\partial r}\right] \\
& \frac{1}{r} \frac{\partial \psi}{\partial r} \frac{\partial T}{\partial x}-\frac{1}{r} \frac{\partial \psi}{\partial r} \frac{\partial T}{\partial r}=\frac{1}{r} \frac{\partial}{\partial r}\left(r \frac{\partial T}{\partial r}\right) \\
& L e\left[\frac{1}{r} \frac{\partial \psi}{\partial r} \frac{\partial C}{\partial x}-\frac{1}{r} \frac{\partial \psi}{\partial r} \frac{\partial C}{\partial r}\right]=\frac{1}{r} \frac{\partial}{\partial r}\left(r \frac{\partial C}{\partial r}\right)
\end{aligned}
$$


$L b\left[\frac{1}{r} \frac{\partial \psi}{\partial r} \frac{\partial n}{\partial x}-\frac{1}{r} \frac{\partial \psi}{\partial r} \frac{\partial n}{\partial r}\right]+P e\left[\frac{\partial}{\partial r}\left(n \frac{\partial C}{\partial r}\right)\right] \frac{1}{x^{2 m-1}}=\frac{1}{r} \frac{\partial}{\partial r}\left(r \frac{\partial n}{\partial r}\right)$

$\frac{\partial \psi}{\partial x}=0, \frac{\partial T}{\partial r}=-q_{w}(x), \frac{\partial C}{\partial r}=-q_{m}(x), \frac{\partial n}{\partial r}=-q_{n}(x)$ at $r=R(x)$

$\frac{1}{r} \frac{\partial \psi}{\partial r}=U_{w}(x), T \rightarrow T_{\infty}, C \rightarrow C_{\infty}, n \rightarrow 0 \quad$ at $r \rightarrow \infty$

Following variables were used for non-dimensionalizing

$$
\begin{aligned}
& x=\frac{\bar{x}}{L}, r=P e^{\frac{1}{2}} \frac{\bar{r}}{L}, u=\frac{\bar{u}}{u_{\infty}}, v=P e^{\frac{1}{2}} \frac{\bar{v}}{u_{\infty}}, R(x)=P e^{\frac{1}{2}} \frac{\bar{R}(x)}{L}, r=P e^{\frac{1}{2}} \frac{\bar{r}}{L}, U_{w}=\frac{\bar{U}_{w}}{u_{\infty}} \\
& T=\frac{k P e^{\frac{1}{2}}\left(\bar{T}-T_{\infty}\right)}{q_{w} L}, C=\frac{D_{m} P e^{\frac{1}{2}}\left(\bar{C}-C_{\infty}\right)}{q_{m} L}, n=\frac{D_{n} P e^{\frac{1}{2}} \bar{n}}{q_{n} L}, q_{w}=\frac{\bar{q}_{w}}{k}, q_{m}=\frac{\bar{q}_{m}}{D_{m}}, q_{n}=\frac{\bar{q}_{n}}{D_{n}}
\end{aligned}
$$

Where $\mathrm{T}, \mathrm{C}, \mathrm{n}$ are temperature, concentration and volume fraction of motile microorganism. $q_{w}$ is the variable surface heat flux, $q_{m}$ is the variable surface mass flux and $q_{n}$ is the variable surface motile microorganism flux. And also,

Rayleigh number $R a=\frac{g \beta_{T} q_{w} L^{2}}{v \alpha}$, Peclet number $P e=\frac{u_{\infty} L}{\alpha}$, Mixed convection parameter $\lambda=\frac{R a^{\frac{1}{3}}}{P e^{\frac{1}{2}}}$

, Buoyancy parameter $N_{1}=\frac{\beta_{c} q_{m} k}{\beta_{T} q_{w} D_{m}}, N_{2}=\frac{\beta_{n} q_{n} k}{\beta_{T} q_{w} D_{n}}$, Lewis number $L e=\frac{\alpha}{D_{m}}$, Bioconvection

Lewis number $L b=\frac{\alpha}{D_{n}}$, Bioconvection peclet number $P e=\frac{b W_{c}}{D_{n}}$

Now the similarity transformations are

$$
\begin{aligned}
& \eta=x^{m-1} r, \psi=x f(\eta), T=x^{2 m-1} \theta(\eta), C=x^{2 m-1} \phi(\eta), n=x^{2 m-1} \chi(\eta) \\
& U_{w}=x^{2 m-1}, q_{w}=x^{3 m-2}, q_{m}=x^{3 m-2}, q_{n}=x^{3 m-2}
\end{aligned}
$$

Where $\psi$ is the stream function, which is defined in the usual way as

$$
u=\frac{1}{r} \frac{\partial \psi}{\partial y}, v=-\frac{1}{r} \frac{\partial \psi}{\partial x}
$$

Setting $\eta=a$ the relationship $\eta=\frac{r}{x^{1-m}}$ describes the shape and size of the body considered with its surface assumed by

$$
R(x)=a x^{1-m}
$$

Here for $\mathrm{m}=1$ corresponds to a cylinder, $\mathrm{m}=0.5$ corresponds to a paraboloid (blunt nosed configuration), $\mathrm{m}=0$ corresponds to a cone. 
Now the transformed ordinary differential equations are

$$
\begin{aligned}
& f^{\prime \prime}=(\lambda)^{3} \eta\left[\theta^{\prime}+N_{1} \phi^{\prime}+N_{2} \chi^{\prime}\right] \\
& \eta \theta^{\prime \prime}+\theta^{\prime}+f \theta^{\prime}-(2 m-1) f^{\prime} \theta=0 \\
& \eta \varphi^{\prime \prime}+\varphi^{\prime}+\text { Le.f} f \varphi^{\prime}-(2 m-1) L e . f^{\prime} \phi=0 \\
& \eta \chi^{\prime \prime}+\chi^{\prime}+L b . f \chi^{\prime}-L b .(2 m-1) f^{\prime} \chi-P e\left(\eta \phi^{\prime} \chi^{\prime}+\chi \varphi^{\prime}+\eta \phi^{\prime \prime}\right)=0
\end{aligned}
$$

The transformed boundary conditions become

$\eta=a, f(a)=0, \theta^{\prime}(a)=-1, \varphi^{\prime}(a)=-1, \chi^{\prime}(a)=-1$ and

$\eta \rightarrow \infty, f^{\prime} \rightarrow a, \theta \rightarrow 0, \varphi \rightarrow 0, \chi \rightarrow 0$

The surface temperature $T_{w}$, surface fluid concentration $C_{w}$ and surface motile microorganism concentration $n_{w}$ are defined as

$T_{w}=\frac{k P e^{\frac{1}{2}}\left(\bar{T}_{w}-T_{\infty}\right)}{q_{w} L}, C_{w}=\frac{D_{m} P e^{\frac{1}{2}}\left(\bar{C}_{w}-C_{\infty}\right)}{q_{m} L}, n_{w}=\frac{D_{n} P e^{\frac{1}{2}} \bar{n}_{w}}{q_{n} L}$

Using non-dimensional transformations and similarity transformations finally we can get,

$T=x^{2 m-1} \theta(a), C=x^{2 m-1} \phi(a), n=x^{2 m-1} \chi(a)$

\section{Numerical Method}

Simulation of the transformed Eqns. (6-9) subject to the boundary conditions (10)-(11) are found with the help of Matlab BVP4C numerical method for various values of the flow controlling parameters. In the context of bvp4c function described above we need to transform the governing equations into first order differential equation. At first Equations (6-9) can be rearranged in the following way

$$
\begin{aligned}
& f^{\prime \prime}=\lambda^{3} \eta\left[\theta^{\prime}+N_{1} \phi^{\prime}+N_{2} \chi^{\prime}\right] \\
& \theta^{\prime \prime}=\frac{-\theta^{\prime}-f \theta^{\prime}+(2 m-1) f^{\prime} \theta}{\eta} \\
& \phi^{\prime \prime}=\frac{-\phi^{\prime}-L e f \phi^{\prime}+(2 m-1) L e f^{\prime} \phi}{\eta} \\
& \chi^{\prime \prime}=\frac{-\chi^{\prime}-L b f \chi^{\prime}+(2 m-1) L b f^{\prime} \chi+P e\left(\eta \phi^{\prime} \chi^{\prime}+\chi \phi^{\prime}+\eta \phi^{\prime \prime}\right)}{\eta}
\end{aligned}
$$


Now we need to transform this above equation into first order differential equation. For this let $\eta=$ $a$ and

$$
\begin{gathered}
y_{1}=f, \quad y_{2}=f^{\prime} \\
y_{3}=\theta, \quad y_{4}=\theta^{\prime}, \quad y_{5}=\phi, \\
y_{6}=\phi^{\prime}, \quad y_{7}=\chi, \quad y_{8}=\chi^{\prime}
\end{gathered}
$$

The corresponding first order differential equations are

$$
\begin{aligned}
\frac{d y_{1}}{d x} & =f^{\prime}=y_{2} \\
\frac{d y_{2}}{d x} & =f^{\prime \prime}=\lambda^{3} a\left[y_{4}+y_{6} N_{1}+y_{8} N_{2}\right] \\
\frac{d y_{4}}{d x} & =\theta^{\prime \prime}=\frac{-y_{4}-y_{1} y_{4}+(2 m-1) y_{2} y_{3}}{a} \\
\frac{d y_{6}}{d x} & =\phi^{\prime \prime}=\frac{-y_{6}-y_{1} y_{6} L e+(2 m-1) y_{2} y_{5} L e}{a} \\
\frac{d y_{8}}{d x} & =\chi^{\prime \prime}=\frac{-y_{8}-y_{1} y_{8} L b+(2 m-1) y_{2} y_{8} L b+\operatorname{Pe}\left(a y_{6} y_{8}+y_{7} y_{6}+y_{7}\left(-y_{6}-\operatorname{Ley}_{1} y_{6}+(2 m-1) \operatorname{Ley}_{2} y_{5}\right)\right)}{a}
\end{aligned}
$$

The boundary conditions become considering ya be the left boundary, $y b$ be the right boundary

$$
\begin{aligned}
& y(a)=0, y(b)-a=0 \\
& y a(4)+1=0, y b(3)=0 \\
& y a(6)+1=0, y b(5)=0 \\
& y a(8)+1=0, y b(7)=0
\end{aligned}
$$

For the validation of the results the differential equations are again solved numerically using Maple 14.0 with the help of dsolve command where asymptotic boundary conditions (10)-(11) are replaced by using a value of 5 for the similarity variable $\eta_{\max }=5$. The obtaining results for both cases as shown in Table 1. show good agreement and exactness of numerical calculations. 
Table 1. Comparison Results of $f^{\prime}(a), \theta_{w}(a), \phi_{w}(a), \chi_{w}(a)$

when $\lambda=0.5, N_{1}=0.0, N_{2}=0.0, L e=0.0, L b=0.0, P e=0.0$

\begin{tabular}{|l|l|l|l|l|l|l|l|l|l|}
\hline $\mathrm{a}$ & $\mathrm{m}$ & $\begin{array}{l}f^{\prime}(a) \\
(\text { Matlab } \\
\text { Bvp4c) }\end{array}$ & $\begin{array}{l}f^{\prime}(a) \\
(\text { Maple } \\
14.0)\end{array}$ & $\begin{array}{l}\theta_{w}(a) \\
(\text { Matlab } \\
\text { Bvp4c) }\end{array}$ & $\begin{array}{l}\theta_{w}(a) \\
(\text { Maple } \\
14.0)\end{array}$ & $\begin{array}{l}\phi_{w}(a) \\
\text { (Matlab } \\
\text { Bvp4c) }\end{array}$ & $\begin{array}{l}\phi_{w}(a) \\
\text { (Maple } \\
14.0)\end{array}$ & $\begin{array}{l}\chi_{w}(a) \\
\text { (Matlab } \\
\text { Bvp4c) }\end{array}$ & $\begin{array}{l}\chi_{w}(a) \\
(\text { Maple } \\
14.0)\end{array}$ \\
\hline 0.1 & 0.5 & 0.146406 & 0.146406 & 0.346817 & 0.346824 & 0.391195 & 0.391202 & 0.391195 & 0.391202 \\
\hline 0.2 & 1.0 & 0.135207 & 0.135206 & 0.298372 & 0.298377 & 0.391199 & 0.391202 & 0.391199 & 0.391202 \\
\hline & 0.5 & 0.276678 & 0.276671 & 0.518732 & 0.518721 & 0.643770 & 0.643775 & 0.643770 & 0.643775 \\
\hline
\end{tabular}

\section{Result Discussion}

Velocity profile distribution for different values of mixed convection parameter $\lambda$ and buoyancy parameter $N_{1}$ are shown in Fig (2-3). Velocity profile increases with the growing values of $\lambda$ and $N_{1}$ because of inciting attitude of buoyancy forces in case of both isothermal $(\mathrm{m}=0.5)$ and nonisothermal $(\mathrm{m}=1.0)$ needle scenarios shown in Fig $(2 \mathrm{a})-(3 \mathrm{a})$ and also for different needle sizes shown in Fig (2b)-(3b). The velocity boundary layer thickness increases for isothermal needle comparing to non-isothermal needle. The velocity profile enhances with the increasing value of needle thickness. The larger contact area of needle surface which causes momentum diffusion enhances flow profile. And also physically, the surface of the needle in contact with the fluid particles decreases when the size of the needle is getting smaller. This causes the friction force that occurs on the needle surface and the fluid flow will be reduced. 


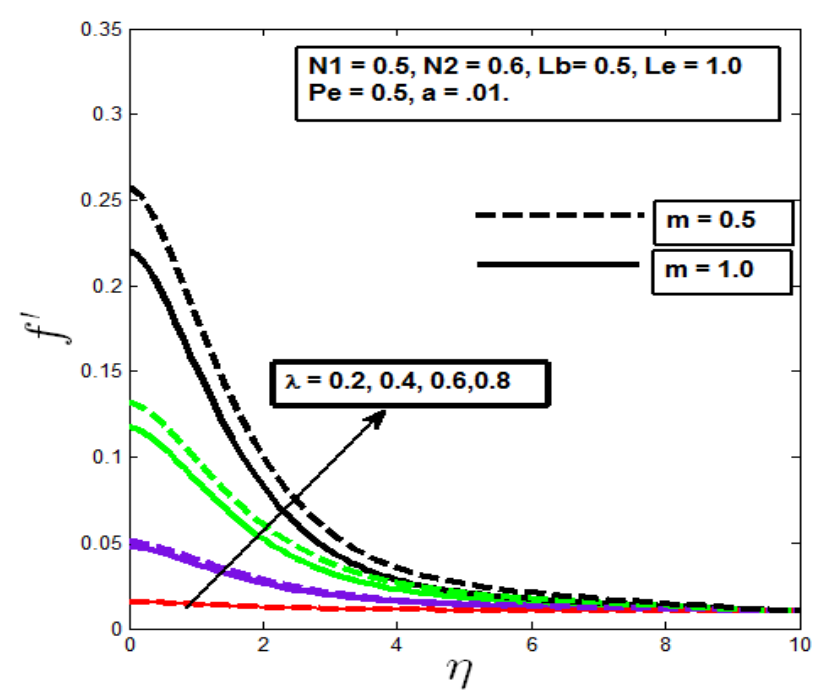

(a)

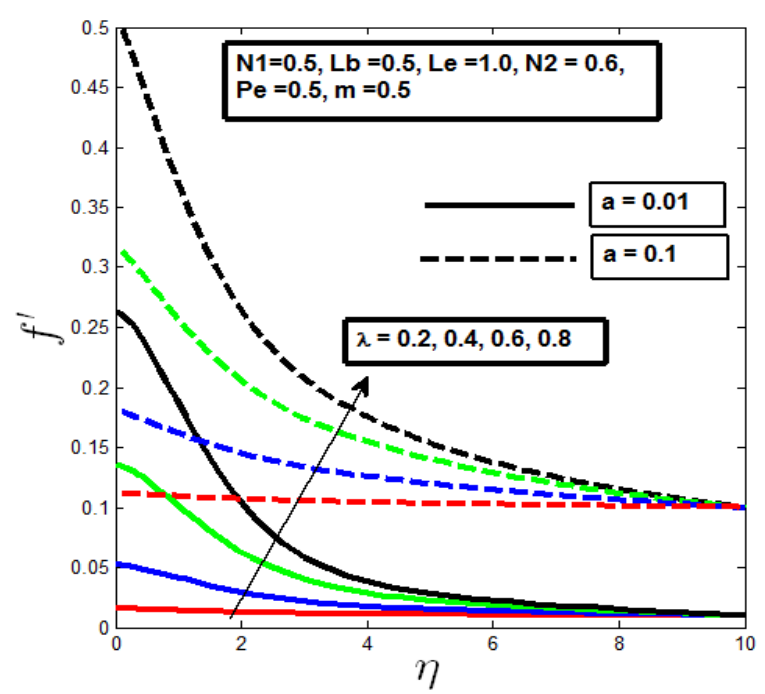

(b)

Fig 2: Velocity profile with variation of mixed convection parameter for a) differential values of $\mathbf{m}$ b) different needle sizes.

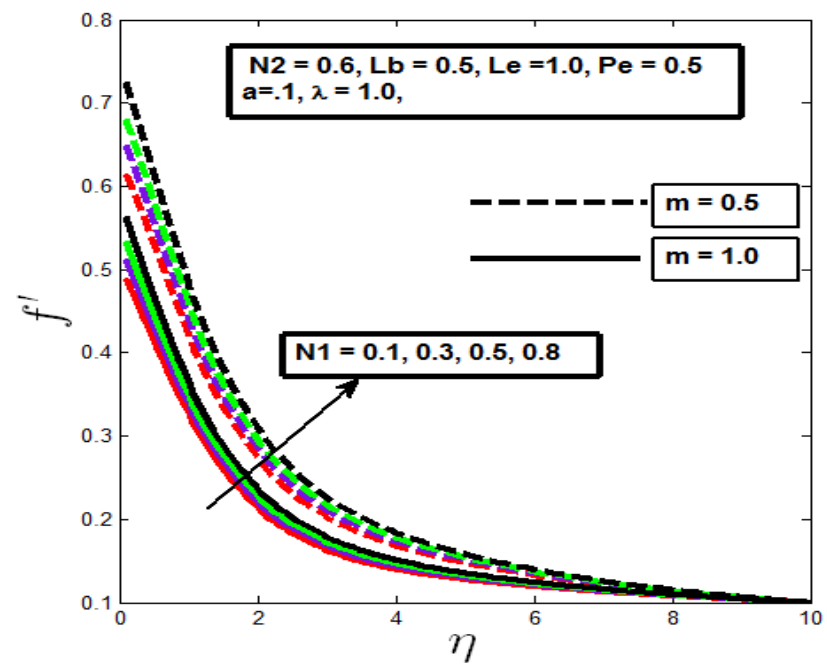

(a)

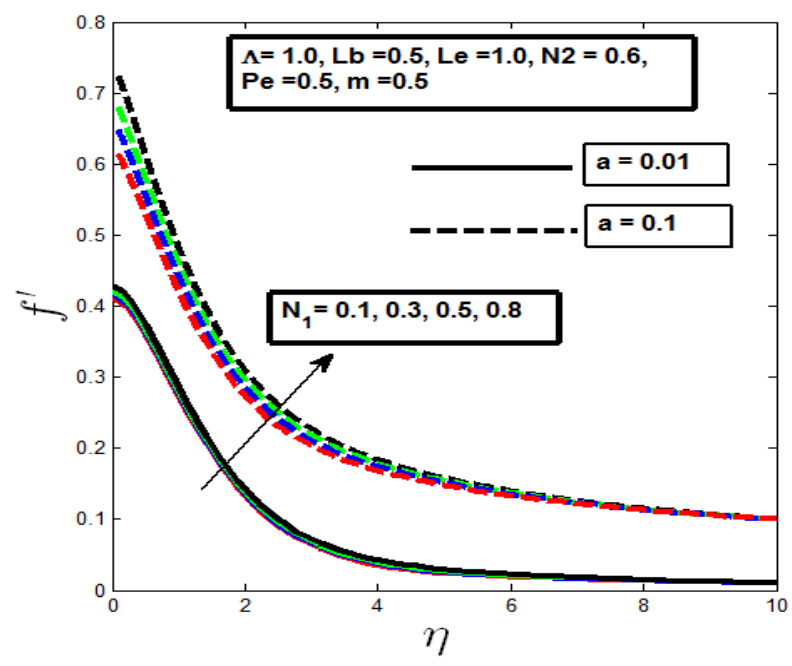

(b)

Fig 3: Velocity profile with variation Buoyancy $\operatorname{parameter} N_{1}$ for a) differential values of $\mathrm{m}$ b) different needle sizes.

In Fig (4-5) Temperature profile reduces with the growing values of mixed convection parameter $\lambda$ and Buoyancy parameter $N_{2}$. Higher reduction of temperature profiles is observed for nonisothermal needle (when $\mathrm{m}=1.0$ ) which is the opposite behavior to the velocity profile. The increment of the thickness of needle leads to a thicker thermal boundary layer which causes lower needle wall temperature and reduces corresponding temperature profiles are shown in Fig (4b)5(b). And also according to [34] the ascension in momentum diffusion rate inhibits the thermal diffusion which induces cooling of the boundary layer and a minimization in thermal boundary layer thickness. 


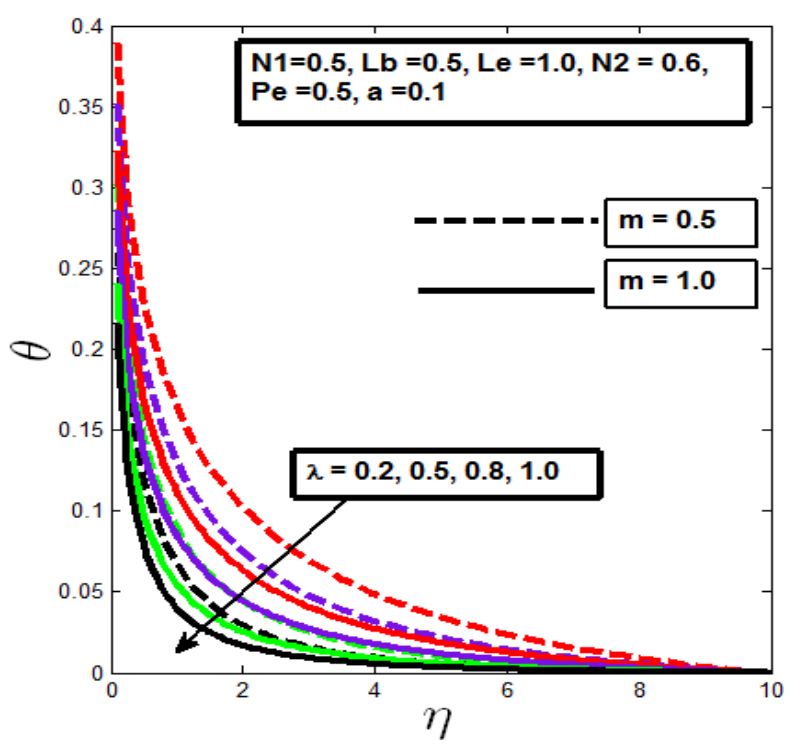

(a)

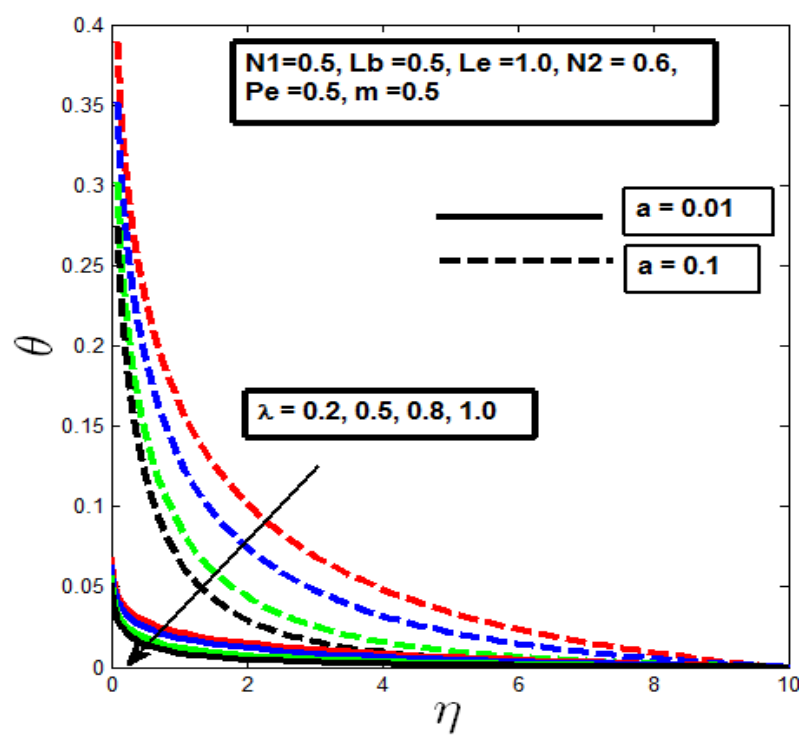

(b)

Fig 4: Effect of mixed convection parameter $\lambda$ on Temperature profile for a) different values of $\mathbf{m} b$ ) different needle sizes

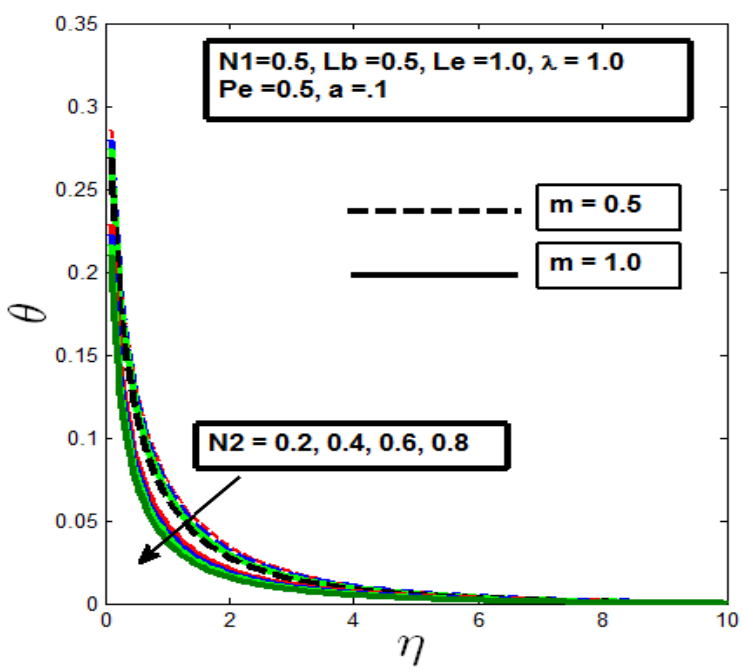

(a)

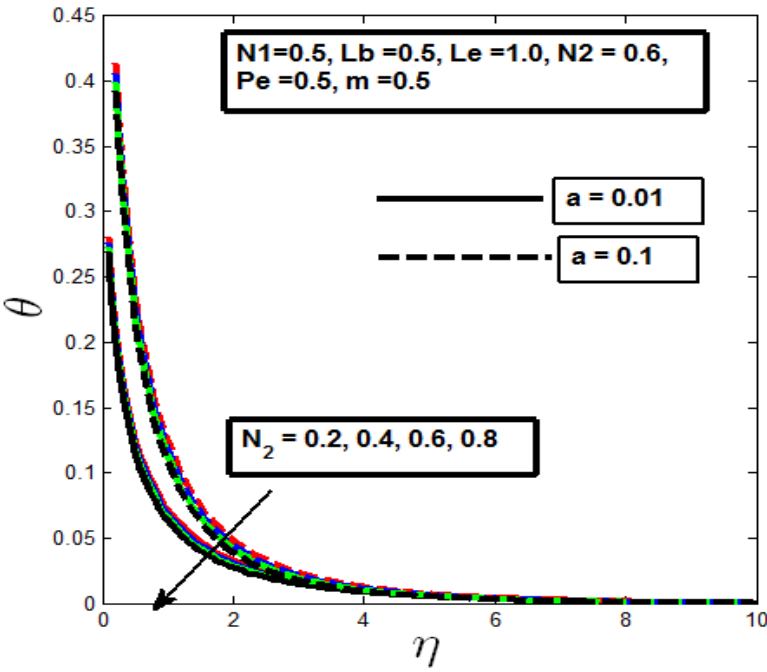

(b)

Fig 5: Temperature profile with variation Buoyancy parameter $N_{2}$ for a) different values of $\mathrm{m} \mathrm{b}$ ) different needle sizes.

Concentration profile decreases with increasing values for mixed convection parameter are shown in Fig 6 where reduction is mostly occurred for non-isothermal case $(\mathrm{m}=1)$ and also it is observed in Fig 6(b) that slender surface of needle reduces concentration profile. The Lewis number Le is defined as the ratio of thermal diffusivity to mass diffusivity which is the prominent factor to study heat and mass transfer. So Lewis parameter Le has a great impact on concentration profile. Increasing values of Le lessen the boundary layer thickness of concentration profile for the isothermal and non -isothermal needles shown in Fig 7. 


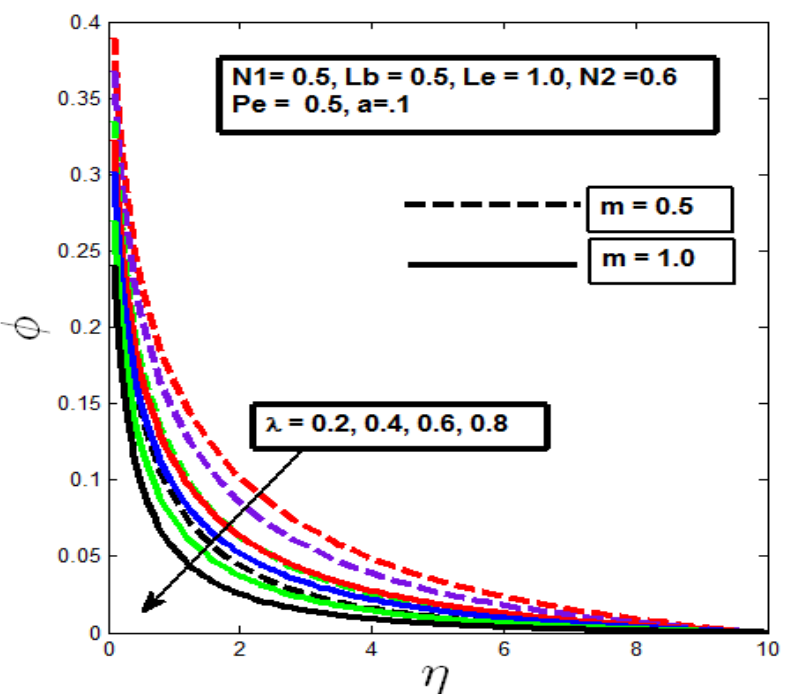

(a)

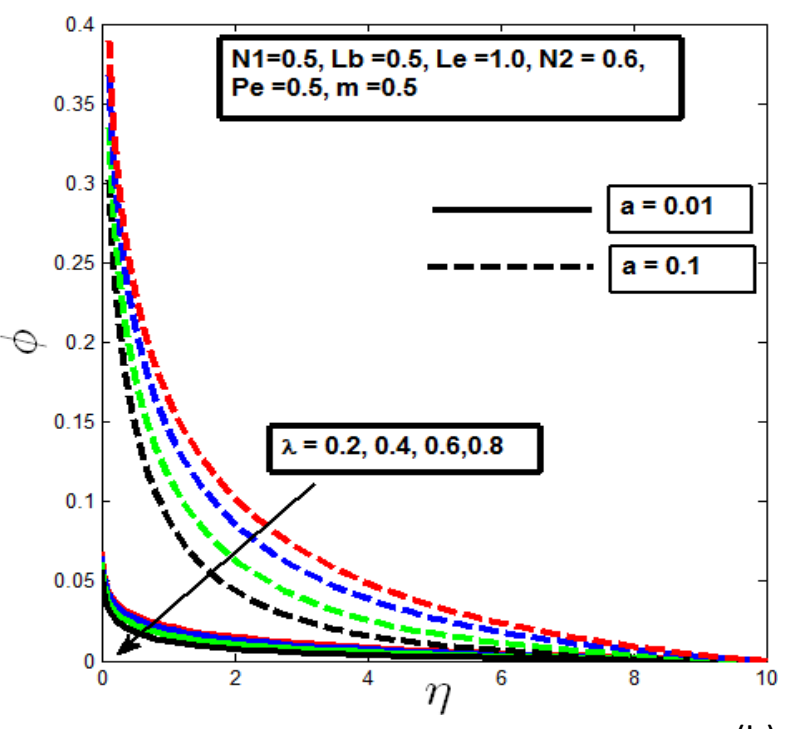

(b)

Fig 6: Concentration profile with variation of mixed convection parameter for a) different values of m b) different needle sizes

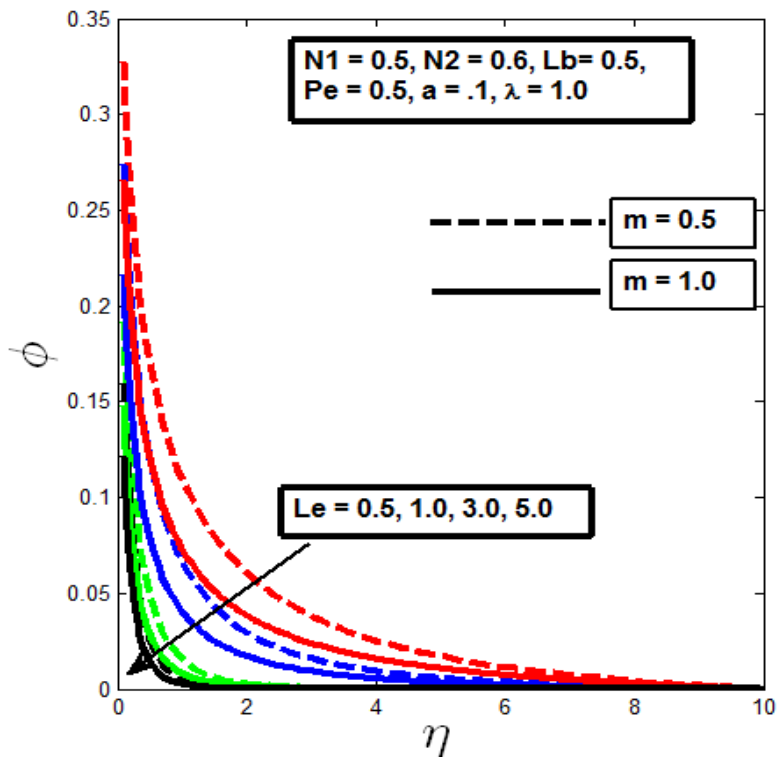

(a)

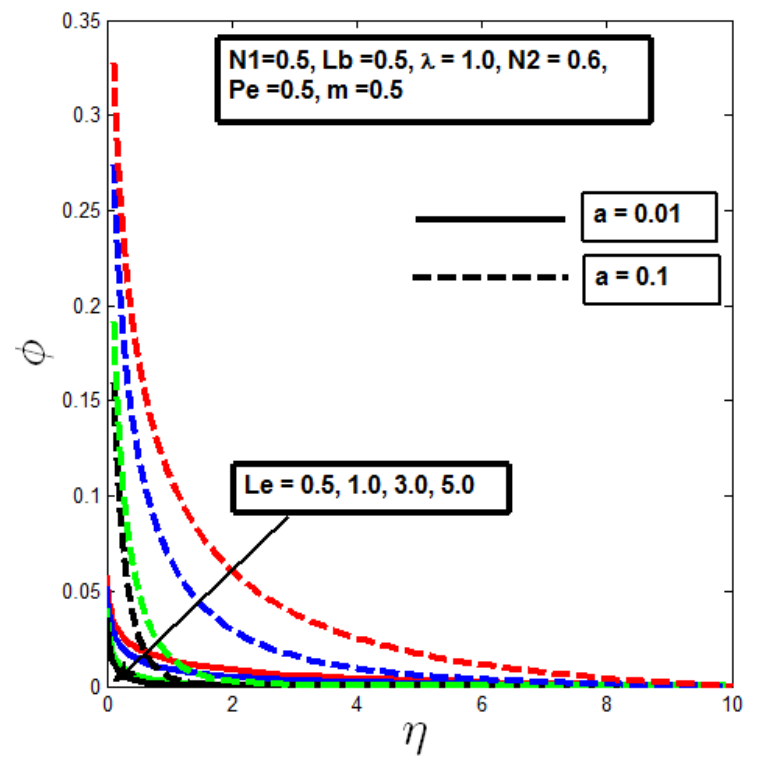

(b)

Fig 7: Concentration profile with variation of Lewis parameter Le for a) different values of $\mathbf{m} b$ ) different needle sizes.

In Fig (8-10) it is observed that microorganism profiles decrease for higher values of mixed convection parameter $\lambda$ and also for Bioconvection Lewis number Lb and Bioconvection peclet number Pe. Bioconvection peclet number Pe and Bioconvection Lewis number Lb rise the mobility of fluid causes the quantity of motile microorganism thickness reduces. It is observed in Fig (8(a)10(a)) that the behavior of microorganism profiles are quite similar for the both isothermal (m $=0.5)$ and non-isothermal $(\mathrm{m}=1.0)$ cases. And also it is noticeable in Fig. 8(b)-10(b) smaller 
dimensions of needle decrease the boundary layer thickness of Microorganism profile. The reason behind these phenomena is the decrement of needle sizes increases motile microorganism transfer rate and decreases boundary layer thickness of microorganism profile.

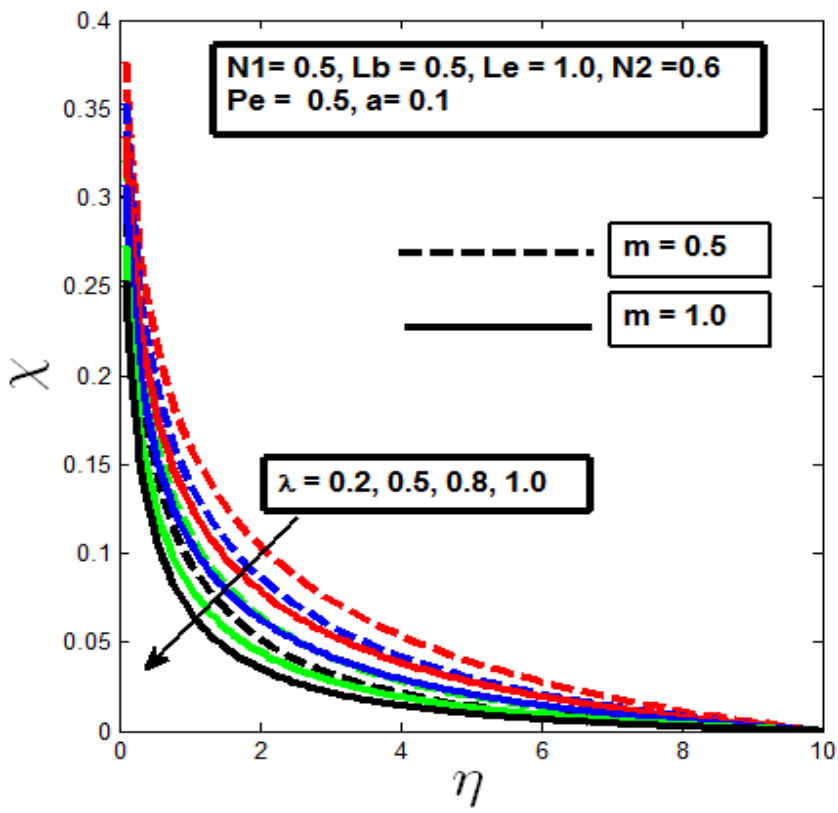

(a)

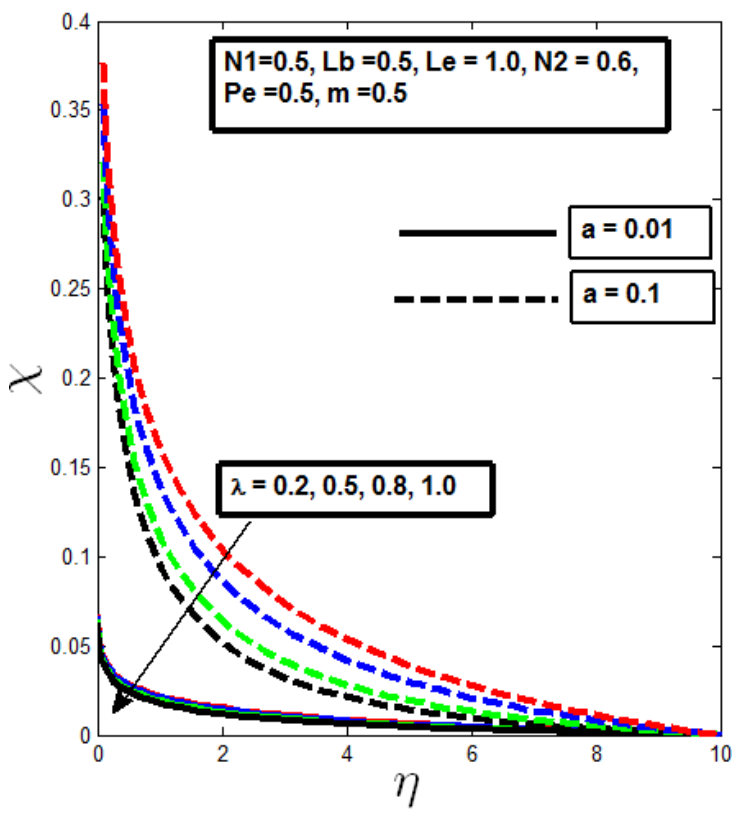

(b)

Fig 8: Microorganism profile with variation of mixed convection parameter for a) different values of $\mathbf{m} \mathbf{b}$ ) different needle sizes

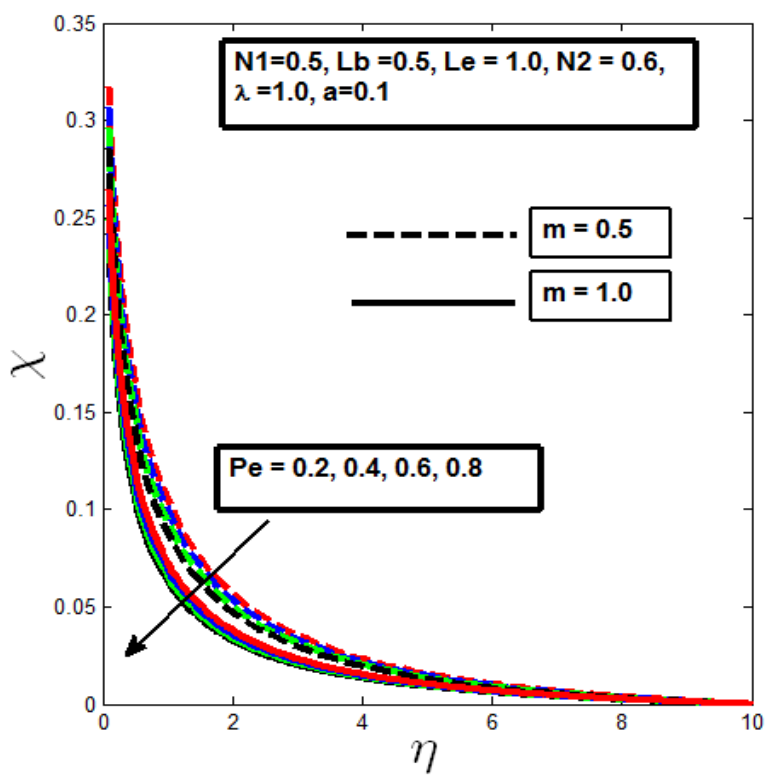

(a)

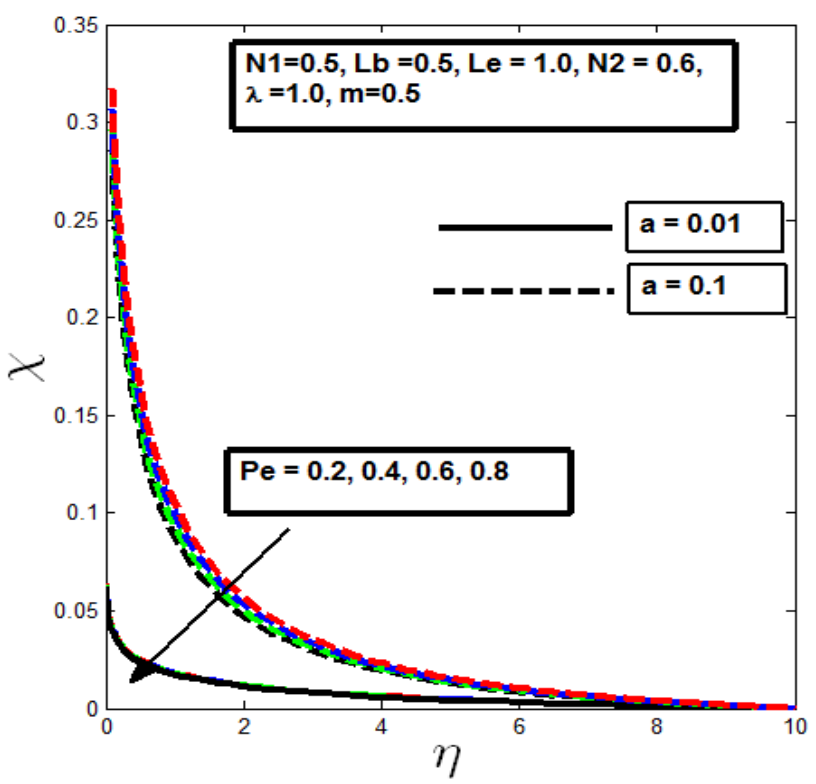

(b)

Fig 9: Microorganism profile with variation of Bioconvection Peclet number for a) different values of $\mathbf{m} \mathbf{b}$ ) different needle sizes 


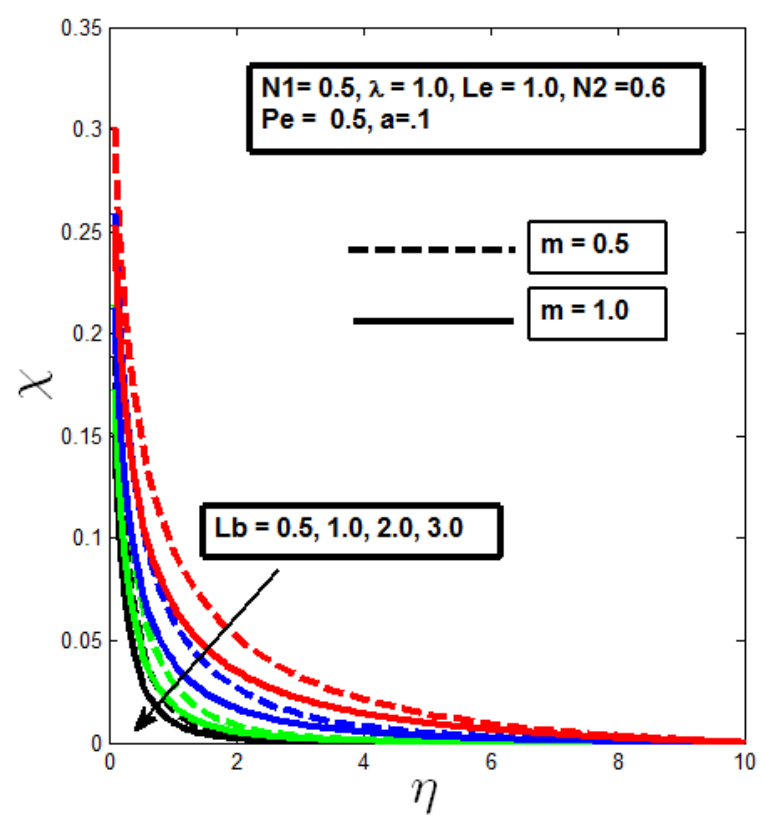

(a)

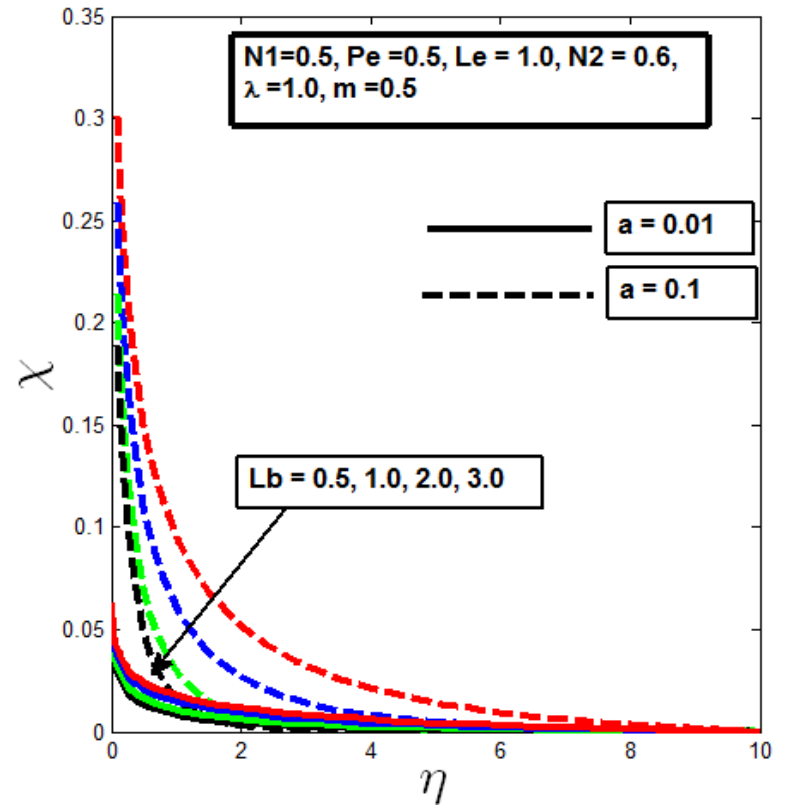

(b)

Fig 10: Microorganism profile with variation Bioconvection Lewis parameter Lb for a) different values of $\mathbf{m} \mathbf{b}$ ) different needle sizes.

Variation of surface temperature, fluid concentration and also surface motile microorganism concentration with mixed convection parameter $\lambda$ are shown in Fig (11-13). Surface temperature, concentration and motile microorganism density decrease with the increasing values of $\lambda$. In all cases surface temperature and concentrations decreases with the increasing values of $m$ which states reduction is higher for non isothermal cases. On the other hand slender needle reduces wall temperature, fluid concentration and also motile microorganism concentration shown in Fig (11$13)$.

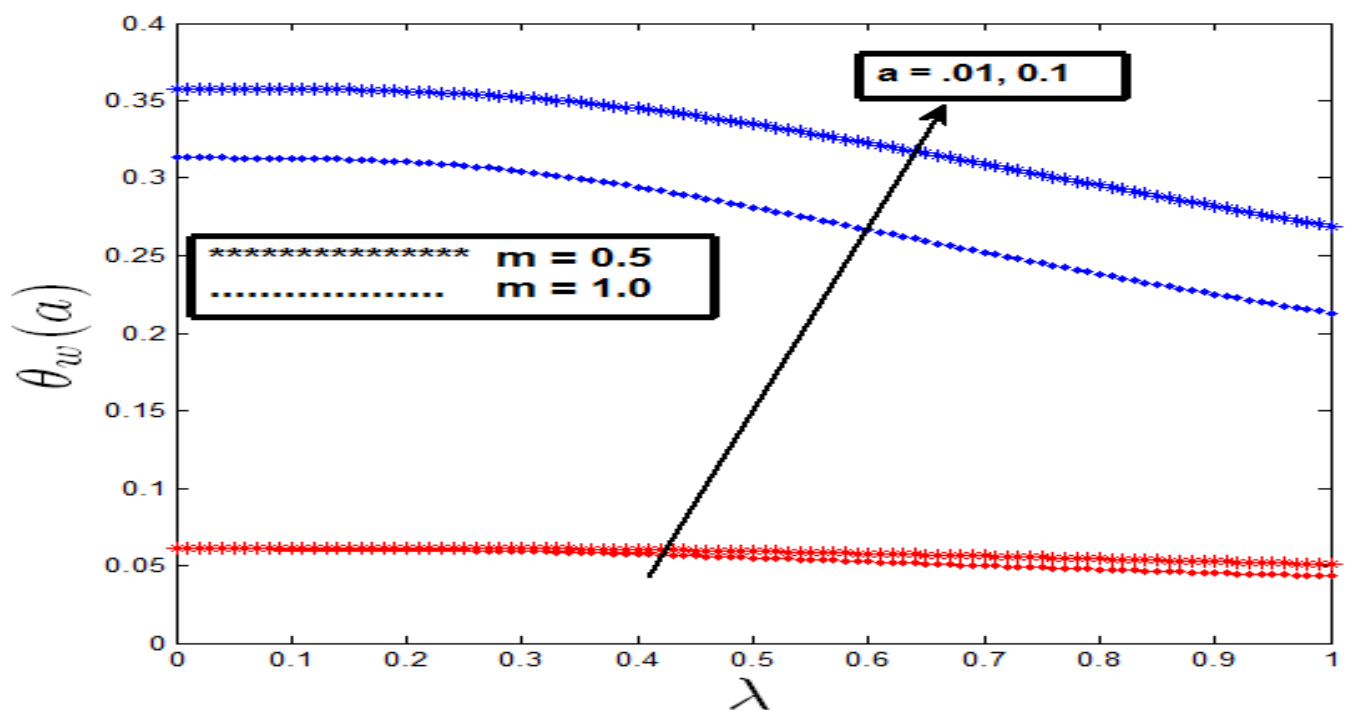

Fig 11. Variation of Surface Temperature with mixed convection parameter $\lambda$. 


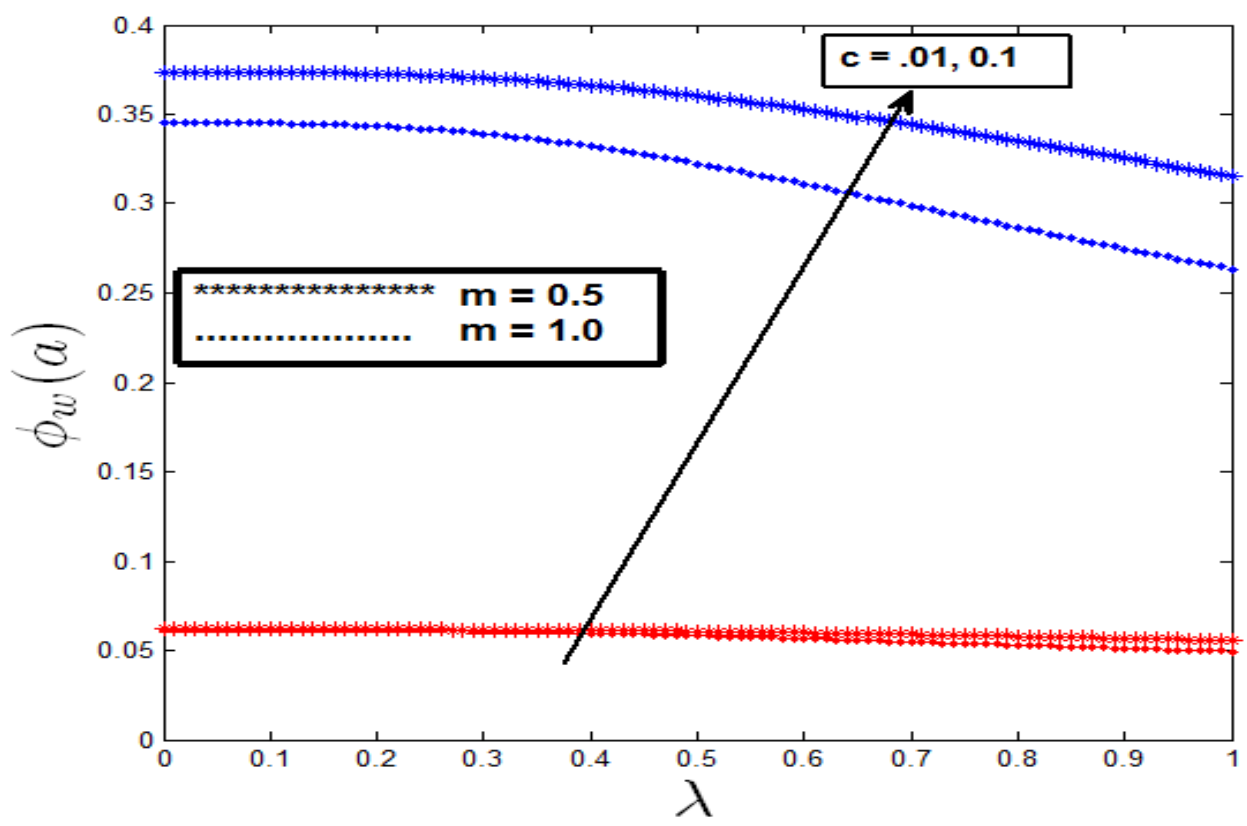

Fig 12. Variation of Surface Fluid Concentration with mixed convection parameter $\lambda$.

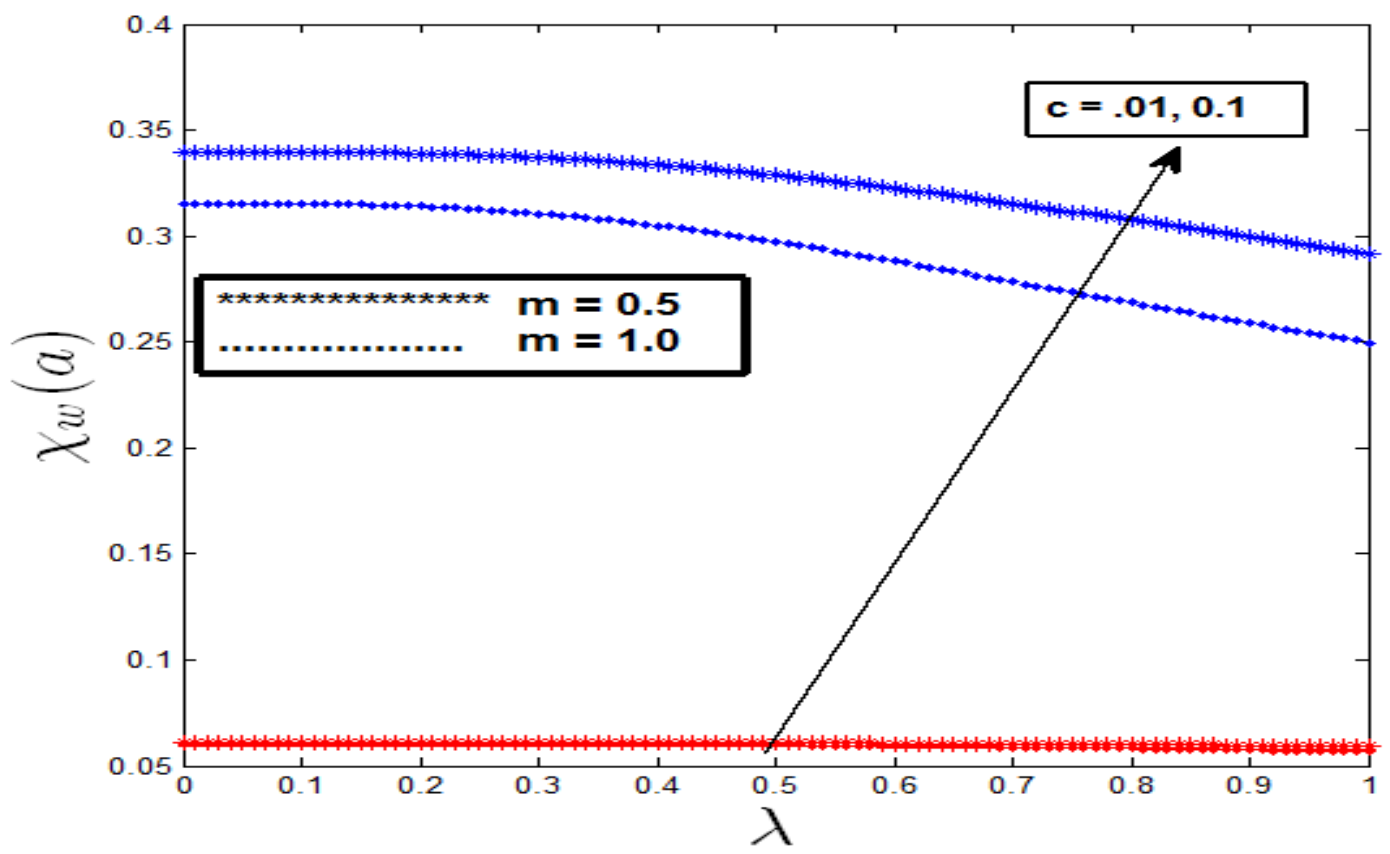

Fig 13. Variation of Surface Motile microorganism Concentration with mixed convection parameter $\lambda$. 


\section{Conclusion}

A new mathematical model for steady two-dimensional mixed convection flow in the presence of gyrotactic microorganism with variable surface heat, mass and motile microorganism flux is studied theoretically and numerically. The influences of isothermal, non -isothermal needle with different sizes have been taken into consideration. The effects of governing parameter on the flow, heat, mass and microorganism characteristic are discussed. The key findings of this analysis can be summarized as follows:

- Velocity profile increases with the increasing values of mixed convection parameter $\lambda$ where temperature, concentration and microorganism profile decreases.

- Buoyancy parameters enhance velocity profiles and reduce temperature profile.

- Lewis parameter Le has great influence on concentration profile which reduces boundary layer thickness of concentration profile.

- Boundary layer thickness of microorganism profile reduces with bioconvection Lewis number Lb and bioconvection peclet number Pe.

- All the physical quantities reduce with non-isothermal power law index.

- Velocity profile increases with the increasing value of needle thickness. Temperature concentration and microorganism profiles decrease for slender needle.

\section{References}

[1] Nasir, N.A.A.M., Ishak, A. \&Pop, I. Stagnation point flow and heat transfer past a permeable quadratically stretching /shrinking sheet. Chin. J. Phys. 55, 2081- 2091(2017).

[2] Tamim, H., Dinar, Saeed,D., Hosseini, R., Khalili, S. \& Pop, I. Unsteady mixed convection flow of a nanofluid near orthogonal stagnation point on a vertical permeable surface. $P$ I MECH ENG E-J PRO. 228(3), 226-237(2014).

[3] Grosan, T. \& Pop, I. Axisymmetric mixed convection boundary layer flow past a vertical cylinder in a nanofluid. Int. J. Heat Mass Transf. 54, 3139- 3145(2011).

[4] Seshadri, R. \& Munjam, S. R. Mixed convection flow due to a vertical plate in the presence of heat source and chemical reaction. Ain Shams Engineering Journal. 7(2), 671-682(2016).

[5] Rosali,H., Ishak,A., Nazar, R. \& Pop,I. Mixed convection boundary layer flow past a vertical cone embedded in a porous medium subjected to a convective boundary condition. Propulsion and Power Research. 5(2), 118-122(2016).

[6] Liao, C.C \& Lin, C. Mixed convection of a heated rotating cylinder in a square enclosure, International Journal of Heat and Mass Transfer. 72, 9-22(2014).

[7] Aly, A. M., \& Raizah, Z. A. S. Mixed Convection in an Inclined Nanofluid Filled-Cavity Saturated With a Partially Layered Porous Medium." ASME. J. Thermal Sci. Eng. Appl. 11(4): 041002 (2019). 
[8] Dutta, B. K., Roy, P. \& Gupta, A.S. Temperature field in flow over a stretching sheet with uniform heat flux. International Communications in Heat and Mass Transfer, 12(1),89-94(1985).

[9] Chang, C.-L. \& Lee, Z.-Y.Free convection on a vertical plate with uniform and constant heat flux in a thermally stratified micropolar fluid. Mechanics Research Communications, 35(6).421427(2008).

[10] Cheng, C.-Y. Soret and Dufour effects on natural convection boundary layer flow over a vertical cone in a porous medium with constant wall heat and mass fluxes. International Communications in Heat and Mass Transfer, 38(1).44-48(2011).

[11] Elbashbeshy, E.M.A. \& Bazid, M.A. The mixed convection along a vertical plate with variable surface heat flux embedded in porous medium. Applied Mathematics and Computation, 125(2-3), 317-324(2002).

[12] Ishak, A. Mixed convection boundary layer flow over a vertical cylinder with prescribed surface heat flux. J. Phys. A Math. Theor. 42 (19), 8(2009).

[13] Roslinda,N., Amin, N. and Ioan Pop. "Mixed convection boundary layer flow from a sphere with a constant surface heat flux in a micropolar fluid." Journal of Energy Heat and Mass Transfer 24, no. 3 (2002): 195-212.

[14] Ibrahim, W. \& Zemedu, C. MHD Nonlinear Mixed Convection Flow of Micropolar Nanofluid over Nonisothermal Sphere. Mathematical Problems in Engineering. 2020, Article ID 3596368, 20 pages, 2020.

[15] Ahmed, M. M., Nourhan, I. G., Reddy, M.G. \& El-Khatib, M. Magnetohydrodynamic Fluid Flow due to an Unsteady Stretching Sheet with Thermal Radiation, Porous Medium, and Variable Heat Flux. Advances in Astronomy, 2021, Article ID 6686883, 9 pages, 2021.

[16] Alloui, Z., Nguyen, T. H. \& Bilgen, E. Numerical Investigation of thermo-Bioconvection in a suspension of Gravitactic Microorganisms. Int. J. Heat Mass Transf. 56:1435-1441(2007).

[17] Avramenko, A. A. \& Kuznetsov, A. V. The onset of Bio-thermal convection in a suspension of Gyrotactic Microorganisms in a Fluid Layer with an Inclined Temperature Gradient. Int. J. Num. Methods Heat Fluid Flow. 20(1):111-129(2010).

[18] Khan, W.A., Uddin,M.J. \& Ismail, I.A. Free convection of non-Newtonian nano- fluids in Porous media with gyrotactic microorganisms. Transp Porous Med. 97:241-52(2013).

[19] Kuznetsov, A.V. The onset of Thermo-Bioconvection in a shallow Fluid Saturated porous Layer Heated from Below in a suspension of oxytactic microorganisms. Eur. J. Mech. B/ Fluids 25(2):223-233(2006).

[20] Kuznetsov, A.V. Bio-thermal convection induced by two different species of 
Microorganism. Intl. commun. Heat Mass Transf 38(5):548-553(2011).

[21] Kuznetsov, A.V. The onset of nanofluid bioconvection in a suspension containing both nanoparticles and gyrotactic microorganisms. Int J Heat Mass Transf. 37(10):1421-1425 (2010).

[22] Khan, Noor Saeed., Taza Gul, Muhammad Altaf Khan, Ebenezer Bonyah and Saeed Islam. Mixed Convection in gravity driven thin film non-newtonian nanofluids flow with gyrotactic microorganisms. Result in physics $7: 4033-4049(2017)$.

[23] Mahdy, A. Gyrotactic Microorganisms Mixed Convection Nanofluid Flow along an Isothermal Vertical wedge in porous Media. International Journal of Aerospace and Mechanical Engineering 11(4):841-850(2017).

[24] Khan, Waqar A., A.M. Rashad, M.M.M. Abdou and I. Tlili. Natural bioconvection flow of a nanofluid containing gyrotactic microorganisms about a truncated cone. European Journal of Mechanics - B/Fluids 75: 133-142 (2019).

[25] Saleem S, Hunza Rafiq,A. Al-Qahtani, Mohamed Abd El-Aziz, M.Y. Malik and I. L. Animasaun .Magneto Jeffrey Nanofluid Bioconvection over a Rotating Vertical Cone due to Gyrotactic Microorganisms.Mathematical Problems in Engineering 2019, Article ID: 3478037 :11 pages (2019).

[26] Sudnagar, P., Peri K. Kameswaran and B. Rushi Kumar. Gyrotactic Microorganism effects on mixed convective nanofluid flow past a vertical cylinder. Journal of thermal Science and Engineering Applications 11(4): 11 pages (2019).

[27] Mallikarjuna, B., A. Rashad, Ali J. Chamkha,and M. M .M. Abdou. Mixed Convection flow of a nanofluid containing Gyrotactic Microorganisms past a vertical slender cylinder. Front. Heat Mass Transfer 10 : Corpus ID: 126098544(2018).

[28] Rashad, A .M. and Hossain A. Nabwey. Gyrotactic mixed convection flow of a nanofluid past a circular cylinder with convective boundary condition. Journal of the Taiwan Instititute of chemical Engineerings 99:9-17(2019).

[29] Uddin, M.J., Kabir, M.N. \& O. Anwar B’eg, Computational investigation of Stefan blowing and multiple-slip effects on buoyancy-driven bioconvection nanofluid flow with Microorganisms. Int. J. Heat Mass Tran. 95 :116-130 (2016).

[30] Mahdy, A. and Hossam A. Nabwey.'Microorganisms' time-mixed convection nanofluid flow by the stagnation domain of an impulsively rotating sphere due to Newtonian heating,"Results in Physics 19:103347(2020).

[31] Mahdy, A. Unsteady Mixed Bioconvection Flow of Eyring-Powell Nanofluid with Motile Gyrotactic Microorganisms Past Stretching Surface. BioNanoSci. (2021). 
[32] Waqas, H,. Manzoor,U., Shah, Z., Arif, M. \& Shutaywi, M. Magneto-Burgers Nanofluid Stratified Flow with Swimming Motile Microorganisms and Dual Variables Conductivity Configured by a Stretching Cylinder/Plate. Mathematical Problems in Engineering 2021, Article ID 8817435:16 pages (2021).

[33] Amirsom, N.A., Uddin, M.J. \& Ismail, A.I.M. MHD boundary layer bioconvection nonNewtonian flow past a needle with Stefan blowing. Heat. Trans. Asian Res. 48(2): 727-743 (2019).

[34]Beg, 0. A, Zohra, F.T, Uddin, M.J, Ismail, and A.I.M. \& Sathasivam, S. Energy conservation of nanofluids from a biomagnetic needle in the presence of Stefan blowing: Lie symmetry and numerical simulation, Case Studies in Thermal Engineering 24, (100861), (2021).

[35] Salleh, S.N.A., Bachok, N., Arifin, N.M. \& Ali, F.M. Influence of Soret and Dufour on forced convection flow towards a moving thin needle considering Buongiorno's nanofluid model. Alexandria Engineering Journal 59:3897-3906(2020)

[36]Sulochana, C., Ashwinkumar G.P., Sandeep, N. Joule heating effect on a continuously moving thin needle in MHD Sakiadis flow with thermophoresis and Brownian moment. The European Physical Journal Plus 132 :387 (2017).

[37] Lai, F.C., Choi, C.Y. \& Kulacki, F.A. Coupled heat and mass transfer by natural convection from slender bodies of revolution in porous media. Int. Commun. Heat Mass Tran. 17 609-620 (1990).

[38] Cebeci, T., Na, T.Y. Laminar free-convection heat transfer from a needle. Phys. Fluids. 12:463-465(1969).

[39] Narain, J.P., Uberoi, M.S. Combined forced and free-convection heat transfer from vertical thin needles in a uniform stream. Phys. Fluids. 15:1879-1882(1972).

[40] Narain, J.P., Uberoi, M.S. Combined forced and free-convection over thin needles. Int. J. Heat. Mass .Transf. 16:1505-1511(1973).

[41] Chen, J.L.S. Natural convection from needles with variable wall heat flux. J. Heat. Transf. 105:403-406(1983).

[42] Chen, J.L.S. Mixed convection flow about slender bodies of revolution. J. Heat. Transf. 109:1033-1036(1987).

[43] Wang, C.Y. Free convection plume from the tip of a vertical needle. Mech. Res. Commun. 16:95-101(1989).

[44] Wang, C.Y. Mixed convection on a vertical needle with heated tip. Phys. Fluids. A. 2:622625(1990). 
[45] Grosan, T. \& Pop, I. Forced convection boundary layer flow past nonisothermal thin needles in nanofluid. Heat Transf. 133, 054503(2011).

[46] Soid, S.K., Ishak, A. \& Pop, I. Boundary layer flow past a continuously moving thin needle in a nanofluid. Appl. Therm. Eng. 114, 58-64(2017).

[47] Hayat, T., Khan, M.I., M. Farooq, T. \& Yasmeen, A. Alsaedi. Water-carbon nanofluid flow with variable heat flux by a thin needle. J. Mol. Liq. 224:786-791(2016).

[48] R. Ahmad, M.S. Mustafa, Hina, Buongiorno's model for fluid flow around a moving thin needle in a flowing nanofluid: A numerical study. Chin. J. Phys. 55:1264-1274(2017).

[49] Salleh, S.N.A., Bachok, N., Arifin, N.M. \& Ali, F.M. Numerical analysis of boundary layer flow adjacent to a thin needle in nanofluid with the presence of heat source and chemical reaction. Symmetry 11 : 543(2019).

[50] Waini, I., Ishak, A. \& Pop, I. Hybrid Nanofluid Flow Past a Permeable Moving Thin Needle. Mathematics 8:612(2020).

[51] Khan, A., Saeed, A., Tassaddiq, A. et al. Bio-convective and chemically reactive hybrid nanofluid flow upon a thin stirring needle with viscous dissipation. Sci Rep 11, 8066 (2021).

[52] Ahmad, S., Arifin, N.M., Nazar, R. et al. Mixed convection boundary layer flow along vertical moving thin needles with variable heat flux. Heat Mass Transfer 44:473 (2008).

[53] Ahmad, S. Arifin, N.M., Nazar, R. \& Pop,I. Mixed convection boundary layer flow along vertical thin needles: Assisting and opposing flows. International Communications in Heat and Mass Transfer 35(2):157-162(2008). 